\title{
Idiopathic CD4 lymphocytopenia: a case of missing, wandering or ineffective T cells
}

\author{
Dimitrios Zonios', Virginia Sheikh² and Irini Sereti*2
}

\begin{abstract}
Idiopathic CD4 lymphocytopenia (ICL) is a presumed heterogenous syndrome with key element low CD4 T-cell counts (below 300/ $\mathrm{mm}^{3}$ ) without evidence of HIV infection or other known immunodeficiency. The etiology, pathogenesis, and management of ICL remain poorly understood and inadequately defined. The clinical presentation can range from serious opportunistic infections to incidentally diagnosed asymptomatic individuals. Cryptococcal and non-tuberculous mycobacterial infections and progressive multifocal leukoencephalopathy are the most significant presenting infections, although the spectrum of opportunistic diseases can be similar to that in patients with lymphopenia and HIV infection. Malignancy is common and related to opportunistic pathogens with an oncogenic potential. Autoimmune diseases are also seen in ICL with an increased incidence. The etiology of ICL is unknown. Mechanisms implicated in CD4 reduction may include decreased production, increased destruction, and tissue sequestration. New distinct genetic defects have been identified in certain patients with ICL, supporting the hypothesis of the lack of a common etiology in this syndrome. The management of ICL is focused on the treatment of opportunistic infections, appropriate prophylactic antibiotics, and close monitoring. In selected patients with life-threatening infections or profound immunodeficiency, strategies to increase T-cell counts or enhance immune function could be considered and have included interleukin-2, interferon-gamma, interleukin-7, and hematopoietic stem cell transplantation. The prognosis is influenced by the accompanying opportunistic infections and may be affected by publication bias of severe cases with unfavorable outcomes. As newer laboratory investigation techniques are being developed and targeted experimental treatments become available, our comprehension and prognosis of this rare syndrome could be significantly improved.
\end{abstract}

Idiopathic CD4 lymphocytopenia (ICL) was described in 1992 as an immunodeficiency syndrome characterized by opportunistic infections and low CD4 T-cell counts in the absence of HIV infection. Despite the 20 years that have elapsed, the clinical spectrum, pathogenesis, and possible treatment for ICL remain obscure. Here, we attempt to summarize the salient features of this condition on the basis of the available literature to date.

\section{Definition}

ICL is defined by a documented absolute CD4 T lymphocyte count of less than 300 cells per cubic millimeter or of less than $20 \%$ of total $\mathrm{T}$ cells on more than one occasion, usually 2 to 3 months apart, without evidence of HIV

*Correspondence: isereti@niaid.nih.gov

2Laboratory of Immunoregulation, National Institute of Allergy and Infectious Diseases, National Institutes of Health, 10 Center Drive, Bethesda, MD 20892, USA Full list of author information is available at the end of the article infection or any defined immunodeficiency or therapy associated with depressed levels of CD4 T cells.

\section{Pathogenesis}

ICL is a condition of unknown etiology and is considered a heterogenous syndrome possibly encompassing different disorders sharing the common feature of reduced circulating CD4 T-cell counts. In that respect, it is no surprise that no uniform theory for the pathogenesis of ICL has been formalized. In this review, we attempt to summarize the most important reports of possible pathogenetic mechanisms and immunologic abnormalities in ICL.

The failure of CD4 T-cell homeostasis in ICL could be attributed to decreased production, increased destruction, tissue sequestration, or any combination of these. Evidence supporting each of these explanations has been reported, although in some studies it is not possible to decipher whether the findings relate to the cause of ICL or are consequences of the lymphopenia itself. Finally, three recent distinct genetic abnormalities have been 
identified, although familial ICL cases were not seen in our cohort and have otherwise been very rare $[1,2]$.

In terms of decreased CD4 T-cell production, decreased bone marrow clonogenic capability with diminished stem cell precursors contributing to CD4 depletion has been described in three relevant studies [3]. Reduction of p56 (Lck) kinase activity in ICL patients' $\mathrm{T}$ cells compared with healthy control donors was observed in another study, emphasizing the critical role of this kinase in the maintenance of the peripheral CD4 T-cell subset [4]. Disturbed thymic T-cell maturation was finally implicated in ICL pathogenesis in another study [5].

In terms of increased CD4 T-cell destruction, one of the initial reports dealing with ICL pathogenesis demonstrated enhanced apoptotic depletion of CD4 T cells [6], possibly associated with overexpression of Fas and Fas ligand [7]. In our large cohort of ICL cases, we described increased activation and cycling of CD4 $\mathrm{T}$ cells, which were inversely correlated with CD4 T-cell numbers, suggesting a compensatory response to lymphopenia [8]. In another report, we demonstrated that CD4 cycling was strongly associated with levels of plasma lipopolysaccharide [9], implying a possible association of microbial products with lymphopenia-induced proliferation. High serum interleukin 7 (IL-7) levels as well as an inverse correlation of CD4 count and IL-7 levels have been described in ICL $[3,10,11]$. The high IL-7 levels possibly represent cytokine accumulation [12] due to decreased IL-7 receptor alpha chain expression on T cells (CD127) [8,9]. This is supported by the fact that IL-7 levels do not correlate with T-cell cycling and inversely correlate with in vitro CD4 T-cell responses to IL-7 as measured by STAT-5 phosphorylation [13]. Specifically, lower levels of STAT-5 phosphorylation after IL-7 stimulation were observed in both CD4 and CD8 T cells from ICL patients compared with controls. In addition, destabilization of $\mathrm{p} 27^{\mathrm{kip} 1}$, a critical step for IL-7-induced T-cell cycling, was decreased in ICL patients compared with controls after in vitro IL-7 stimulation. These data suggest that high serum IL-7 levels do not necessarily represent a compensatory response and that the blunted T-cell response to IL-7 may be further accentuating T-cell apoptosis and lymphopenia. Autoantibodies to CD4 T cells have also been reported in ICL [14] but with no clear insight into the implications of this finding.

The low CD4 T-cell numbers and higher proportion of memory-activated CD4 T cells may lead to restricted pathogen recognition in ICL. Alpha/beta and gamma/ delta T-cell repertoires of three patients with ICL were reportedly highly restricted, giving rise to mono-/ oligoclonal T-cell expansions [15]. In addition, defective production of interferon-gamma (IFN- $\gamma$ ) and tumor necrosis factor (TNF) was seen in two patients with ICL and cryptococcal meningitis [16], showing that functional
T-cell defects can be associated with presenting infections.

T-cell trafficking and tissue distribution had not been previously studied in ICL. In an immunodeficiency syndrome characterized by warts, hypogammaglobulinemia, infections, and myelokathexis (WHIM), mutations of the chemokine receptor CXCR4 leading to neutropenia and immunodeficiency were identified [17]. In a recent study that focused on chemokine receptor CXCR4 [18], patients with ICL had very low to undetectable levels of surface CXCR4 expression with intracellular accumulation of CXCR4 and its ligand, CXCL12, in CD4 T cells in comparison with healthy controls. Impaired CXCR4 expression was seen exclusively in T cells, both naïve and memory subsets, and was restored after overnight rest. In chemotaxis assays, it was shown that $\mathrm{T}$ cells from patients with ICL had impaired chemotactic responses to CXCL12 and normal responses to CXCL8. There was also a slower reemergence of CXCR4 after ligand binding and internalization. In vivo IL-2 administration seemed to restore CXCR4 expression and responses to CXCL12 in three out of four patients treated. This observation has not yet been reproduced by other groups and should be further explored. To date, our group has not found ICL patients with absent CXCR4 expression on $\mathrm{T}$ cells (unpublished observations).

A genetic etiology in patients with ICL was identified in three recent studies. The first study [19] described two novel missense mutations in $R A G 1$ gene related to a mild clinical ICL phenotype in a girl. RAG1 or RAG2 mutations have been responsible for the majority of patients with severe combined immunodeficiency of the T-BSCID phenotype as well as for the Omenn syndrome [19]. It is consequently concluded that $R A G$ mutations can be responsible for clinical presentations spanning from severe immunodeficiencies to an almost normal phenotype, as seen in this patient with ICL.

The second study [1] implicated mutations in the magnesium transporter gene, MAGT1, to an X-linked primary human immunodeficiency with impaired thymic production of CD4 $\mathrm{T}$ cells and circulating $\mathrm{T}$ cells in two brothers with ICL. This novel syndrome, XMEN (X-linked immunodeficiency with magnesium defect, Epstein virus infection and neoplasia), revealed a role for $\mathrm{Mg}^{+}$as an intracellular second messenger.

The third and most recent study [20] described a heterozygous dominant negative missense mutation of the signaling adaptor protein Uncoordinated 119 (Unc119) in a patient with ICL. The mutation disrupts the Unc119-lymphocyte specific kinase (Lck) interaction that is necessary for the T-cell response to T-cell receptor (TCR) stimulation. In fact, there is impairment in both localization and enzymatic activation of Lck. The patient was a 32-year-old woman with ICL and recurrent otitis 
media, shingles, widespread fungal nail infection, oral herpetic lesions, and bronchiolitis obliterans organizing pneumonia.

\section{Clinical features}

The diagnosis of ICL is typically suspected when an opportunistic infection is identified in an otherwise healthy individual. The literature is dominated by such cases and is constantly expanding. The majority of cases report opportunistic infections - most notably cryptococcal or mycobacterial disease and progressive multifocal leukoencephalopathy (PML) - that are normally seen in HIV-infected patients. The detailed listing of all reported infections exceeds the scope and purpose of this review and has been summarized effectively elsewhere $[21,22]$. It is possible that the apparent presenting clinical spectrum of ICL is influenced by publication bias of more serious infections and adverse outcomes. In that respect, our longitudinal study of 39 patients with ICL [8] offers some insight into the incidence of infections and other complications (namely autoimmune disease and malignancy) during a median follow-up period of 49 months. In this study, the three most common infections at presentation were cryptococcal, genital human papilloma virus (HPV), and non-tuberculous mycobacterial infections. As described extensively in the available literature [23], cryptococcal infections were mainly, but not exclusively, pulmonary or central nervous system disease. Human papilloma virus infections were, notably, treatment-resistant or locally spread with or without cervical intraepithelial neoplasia. Most non-tuberculous mycobacterial infections were due to Mycobacterium avium complex (MAC). Other significant infections at presentation or during follow-up were Pneumocystis jiroveci pneumonia (PCP), PML, and Epstein-Barr virus (EBV)-related lymphoproliferative disease leading to Bcell lymphoma. Most importantly, though, there was a minority of ICL patients who were asymptomatic and remained as such for a long period of time with no infections or other disease. It is reasonable to hypothesize, as a result, that there is a subpopulation within ICL with a more favorable outcome that may be underreported in the literature. This has been suggested in the past [24], although asymptomatic CD4 lymphocytopenia may be extremely rare or transient [25]. Other notable infections described are varicella zoster virus infection, histoplasmosis, Candida, toxoplasmosis, aspergillosis, cytomegalovirus, and Leishmania. Recently, a number of cases of ICL-related PML have been reported, signifying the potential gravity of the ICL diagnosis given the ominous prognosis of this infection and the absence of validated anti-viral therapy targeting John Cunningham (JC) virus [26]. A special consideration is warranted for the cases of ICL and tuberculosis since untreated tuberculosis can lower CD4 T-cell counts and effective tuberculosis treatment usually leads to restoration of CD4 lymphocytopenia [27].

Malignancy is also common in ICL, mainly lymphoma, and specifically EBV-related B-cell [8], primary effusion, and central nervous system lymphoma. Other possible malignancies include Kaposi's sarcoma and cervical or perineal neoplasias in the setting of long-term HPV infections [8]. In summary, patients with ICL and HIVinfected individuals with comparable CD4 T-cell counts appear to have a similar pattern of susceptibility to opportunistic pathogens (manifesting as infections or neoplasms).

\section{Diagnosis and differential diagnosis}

Although establishing the presence of lymphocytopenia in an HIV-uninfected patient with an opportunistic infection is relatively straightforward, determining that the patient has ICL is not. The differential diagnosis of ICL is large (Table 1) [22] and this diagnosis remains one of exclusion and requires an extensive immunologic, hematologic, rheumatologic, and infectious disease workup as well as follow-up testing to confirm the persistence of lymphocytopenia. As stated in the syndrome definition, other forms of immunodeficiency have to be excluded and the provisional ICL diagnosis has to be laboratory-confirmed at least twice during a period of 1 to 3 months. A comprehensive immunologic work-up should be applied in order to exclude HIV infection, lymphoma, autoimmune diseases, other forms of immunodeficiency (such as common variable immunodeficiency), and sarcoidosis (Table 1). As mentioned above with tuberculosis, special attention should also be paid to the possibility that the presenting infection or treatment may be the cause of lymphocytopenia.

The relationship between ICL and autoimmune disease is a complicated one. Although lymphocytopenia is a known consequence of some autoimmune diseases such as systemic lupus erythematosus and Sjögren's syndrome $[8,28]$, patients with ICL appear to be at increased risk for autoimmune diseases possibly due to a break of tolerance (lack of self-recognition) that can occur in the setting of lymphopenia-induced T-cell proliferation lymphopenia $[29,30]$. Autoimmune diagnoses described among patients with ICL include antiphospholipid antibody syndrome, psoriasis, autoimmune hemolytic anemia, ulcerative colitis, Grave's disease, vitiligo, autoimmune thyroiditis, Behçet's disease, and vasculitis. The diagnosis of ICL, when autoimmune disease is present, can therefore be challenging since lymphocytopenia might be a feature of autoimmunity itself or ICL may be the underlying cause of autoimmunity. Complicating the assessment further may be the possibility of transient lymphocytopenia related to immunosuppressive therapies. 


\begin{tabular}{ll}
$\begin{array}{l}\text { Table 1. Differential diagnosis of idiopathic CD4 } \\
\text { lymphocytopenia }\end{array}$ \\
\hline Infections \\
HTLV1,2 \\
Mycobacterial infections \\
EBV, CMV \\
Lymphoma \\
Malignancies/hematologic & Aplastic anemia \\
conditions & Myelodysplastic syndrome \\
& Systemic lupus erythematosus \\
Autoimmune & Sjögren's syndrome \\
& Kikuchi disease \\
& Chemotherapy and immunosuppressants \\
Medications & Corticosteroids \\
& XMEN \\
Genetic syndromes & WHIM \\
Unc119 mutation & Sarcoidosis \\
Various & Common variable immunodeficiency \\
\hline
\end{tabular}

CMV, cytomegalovirus; EBV, Epstein-Barr virus; Unc119, Uncoordinated 119; WHIM, warts, hypogammaglobulinemia, infections, and myelokathexis; XMEN, $X$-linked magnesium deficiency with Epstein-Barr virus and neoplasia.

Lymphocytopenia in the setting of Sjögren's syndrome needs to be addressed separately. Soon after ICL was first described, it was noted that patients with Sjögren's syndrome have an increased incidence of low CD4 T-cell numbers which fulfilled ICL criteria but also correlated with anti-SSA antibody positivity [31] and predisposed these patients to lymphoma [28,32]. In a key study designed to identify predictors of lymphoma in a primary Sjögren's syndrome population, CD4 lymphocytopenia and a low CD4/CD8 ratio were strongly associated with non-Hodgkin's lymphoma [33].

Finally, another significant association that may be overlooked is between lymphocytopenia and sarcoidosis. There have been reported cases of the concomitant existence of the two conditions in the past [34], and there is also an increased incidence of opportunistic infections in sarcoidosis (cryptococcosis in particular), especially in corticosteroid-treated patients. Recently, it has been shown that the low CD4 T-cell count in certain patients with sarcoidosis may relate to disease severity [35] and may represent a distinct phenotype with a favorable response to anti-TNF- $\alpha$ treatment [36]. Since it is unclear whether sarcoidosis may coexist with ICL or lymphopenia may be merely a manifestation of severe sarcoidosis, the diagnosis of ICL should be questioned when sarcoidosis is confirmed. Our position is that CD4 lymphopenia in the presence of an established diagnosis (sarcoidosis or Sjögren's syndrome, for example) could be viewed as an extreme phenotype of the underlying condition and not necessarily as coexistence of ICL with another condition.

There is no consensus for diagnostic investigation of possible ICL cases. The diagnostic work-up focused on excluding alternative diagnoses should be dictated by the presenting symptoms, and special attention should be given to signs of rheumatologic, infectious, immunologic, or hematologic abnormality. The investigation should typically consist of lymphocyte flow cytometry, including CD4, CD8, natural killer, and B-cell subsets as well as immunoglobulin levels, HIV 1-2 serology and PCR, HTLV 1 and 2 testing, EBV and cytomegalovirus PCRs, and an autoantibody panel. The presenting opportunistic infection or suggestive signs or symptoms should dictate the infectious disease work-up. Appropriate imaging by computed tomography or positron emission tomography and bone marrow biopsy should be performed if lymphoma is suspected. If clinical and laboratory features of WHIM are observed, T cells should be assessed for CXCR4 expression. Genetic testing should be performed when familial clustering is observed or when the clinical features suggest any of the described genetic syndromes. Functional T-cell assays looking at cytokine production or proliferation in response to antigen or TCR repertoire analysis are of interest to better understand pathogenesis but have not been clearly linked to clinical prognosis or diagnosis.

\section{Treatment}

The treatment of ICL is influenced by the initial clinical presentation. Monitoring of asymptomatic ICL individuals should be regular during the immediate followup period after diagnosis since most opportunistic infections have been described in the first months of follow-up. Although there is no consensus about how often these patients should be followed, a 3- to 6-month interval may suffice in most cases. After the first few years of follow-up, less frequent evaluation could be appropriate. Special attention should be paid in certain populations with a possible adverse outcome. Low CD8

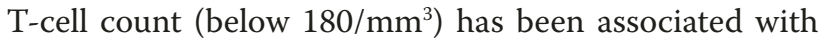
opportunistic infection-related death [23]. Therefore, this subset of ICL individuals might benefit from evaluations that are more frequent. Screening for lymphoma or HPVrelated cervical or anal dysplasia or a combination of these may also be appropriate.

Although it is clear that opportunistic infections in patients with ICL should be treated vigorously with continuing secondary prophylaxis, there is insufficient evidence to guide primary infection prophylaxis. In a median follow-up of 49.5 months in our cohort, infection was diagnosed in 15 (41.6\%) of the patients and severe opportunistic infections were diagnosed in four (13.8\%) 
of these 15 [8]. Interestingly, only one episode of PCP and two of MAC were diagnosed during a cumulative 164 patient-years of follow-up. Our natural history study precluded any firm conclusions about who should be offered prophylaxis but does seem to demonstrate that the risk of MAC and PCP infections is much greater in untreated HIV-infected individuals with AIDS. Although HIV infection and ICL differ substantially in etiology, progression, and prognosis, the literature on prophylaxis of opportunistic diseases in HIV infection provides the best available data with respect to opportunistic infection prophylaxis in ICL. Thus, patients with ICL may reasonably be offered infection prophylaxis that is based on established guidelines for patients with HIV/AIDS.

In selected patients with a history of life-threatening infections or profound immunodeficiency or persistent opportunistic infections, strategies to increase the circulating CD4 lymphocytes could be considered. It is possible that there is publication bias toward reports of successful outcomes of immune-based therapies in this setting. Therapeutic options used to increase CD4 cells or improve immune function (or both) in ICL include the use of IL-2, IFN- $\gamma$ therapy, IL-7, and hematopoietic stem cell transplantation. Therapy with IL-2 was first used in the form of subcutaneous polyethylene glycol-IL-2 for 5.5 years in a woman with chronic severe mycobacterial disease; she had significant clinical improvement but a moderate and slow $\mathrm{CD} 4$ count increase from approximately $29 / \mathrm{mm}^{3}$ before treatment to $100 / \mathrm{mm}^{3}$ after 1 year of therapy [37]. A man with ICL and relapsing generalized herpes zoster infection was treated with IL-2 every other week for 5 months and had clinical improvement and a CD4 count increase from 24 to $93 / \mathrm{mm}^{3}$. The patient developed gastric anaplastic large-cell mucosa-associated lymphoid tissue lymphoma 1 year after IL-2 treatment and subsequently died [22,38]. A 33-year-old woman who had ICL but who also had an IgA and IgG monoclonal gammopathy and renal disease received IL-2 and had a regression of gammopathy and a significant CD4 increase from $40 / \mathrm{mm}^{3}$ to constantly above $300 / \mathrm{mm}^{3}$ after treatment [39]. A 16-year-old boy with ICL and recurrent cryptococcal meningitis was treated with recombinant IL-2 twice weekly and showed clinical improvement and a CD4 count increase from 202 to 262 and $323 / \mathrm{mm}^{3}$ after 5 and 9 months of therapy, respectively [40]. All of the above cases are summarized in a report of a 39-year-old man who had a Mycobacterium avium intracellulare infection that was treated with IL-2 for 35 months and who was apparently cured while his CD4 count increased from 365 to $470 / \mathrm{mm}^{3}$ at the end of therapy [41]. A 64-year-old man with recurrent disseminated Mycobacterium avium intracellulare infection was also treated with IL-2 in three cycles of 5 consecutive days each every other month and had a favorable clinical outcome and CD4 number increases after each IL-2 cycle [42]. Finally, a recent report described a series of four ICL patients who received several courses of recombinant IL-2 for up to 3 years [18]. Three patients responded with either sustained or transient CD4 count increases whereas one did not. Two ICL patients with disseminated MAC were treated with IL-2 at the National Institutes of Health but had no clinical improvement and both subsequently died (Michael Sneller, personal communication).

IFN- $\gamma$ therapy has been offered in selected ICL cases. Three patients with ICL were included in a study of IFN- $\gamma$ in refractory disseminated non-tuberculous mycobacteria infection, and two of them had clinical improvement and CD4 count increases [43]. Two more patients with a potential benefit from IFN- $\gamma$ treatment have been documented in the literature $[16,42]$, whereas others had no clear benefit $[37,38]$.

It has been shown that, in patients with idiopathic CD4 lymphopenia, an impaired IL-7 response to CD4 depletion may contribute to the impaired lymphocyte homeostasis [11]. As such, an increased availability of IL-7 could play a critical role in restoring T-cell homeostasis by enhancing effective proliferation and by improving T-cell survival. IL-7 has been reported to lead to increases in both CD4 and CD8 T cells in other lymphopenic settings at well-tolerated doses [44-46]. IL-7 immunotherapy is currently being investigated in an open-label, single-arm, phase I/IIa clinical trial of glycosylated recombinant human IL-7 (rhIL-7) in ICL patients at risk of disease progression [47]. Successful control of PML has been reported in a patient with ICL by using a combination of CMX001 (investigational oral drug for double-stranded DNA viral diseases, including JC) and IL-7 that led to stabilization of neurologic symptoms and CD4 increases [48].

Finally, it is worth mentioning two key studies of a complete restoration of CD4 counts to normal levels and apparent cure of ICL in individuals treated with nonmyeloablative allogeneic bone marrow transplantation for aplastic anemia or ICL or both [49,50]. Although these results are promising, this therapeutic option clearly should be reserved for a carefully selected population with severe disease in an appropriate clinical setting.

\section{Prognosis}

The prognosis of ICL is critically influenced by the opportunistic infections that may complicate the natural history of the syndrome. Low CD8 numbers $\left(<180 / \mathrm{mm}^{3}\right)$ and the degree of CD4 T-cell activation as measured by HLA-DR expression have been associated with an adverse outcome [8], namely opportunistic infection-related death. Lymphocytopenia is typically not progressive, and in some instances $\mathrm{CD} 4$ numbers can return to normal 
levels in the follow-up period for reasons that are unclear. Finally, as chronic HPV infections may predispose patients to squamous cell carcinomas, appropriate cancer screening should be entertained.

\section{Conclusions}

Despite 20 years of investigation, ICL remains a syndrome of uncertain pathogenesis, prognosis, and treatment and can be a devastating diagnosis for some patients. Although a subset of patients with ICL may remain asymptomatic, others may go on to develop lifethreatening opportunistic infections or autoimmune conditions or severe HPV-related dysplasia and malignancy. The cause or causes of ICL remain unknown, although several distinct genetic mutations have been reported recently and appear to be responsible in some patients. Further study of the genetic basis and immunopathogenesis of this heterogenous condition is required so that targeted treatments for ICL patients at increased risk of significant morbidity and mortality can be safely established.

This article is part of the series on Adult immunodeficiency, edited by Hans-Hartmut Peter. Other articles in this series can be found at http://arthritis-research.com/series/immunodeficiency

\section{Abbreviations}

EBV, Epstein-Barr virus; HPV, human papilloma virus; ICL, idiopathic CD4 Iymphocytopenia; IFN- $\gamma$, interferon-gamma; IL, interleukin; JC, John Cunningham; MAC, Mycobacterium avium complex; PCP, Pneumocystis jiroveci pneumonia; $P C R$, polymerase chain reaction; $P M L$, progressive multifocal leucoencephalopathy; TCR, T-cell receptor; TNF, tumor necrosis factor; WHIM, warts, hypogammaglobulinemia, infections, and myelokathexis.

\section{Competing interests}

The authors declare that they have no competing interests.

\section{Acknowledgments}

The work of VS and IS was supported by the Intramural Research Program of the National Institute of Allergy and Infectious Diseases/National Institutes of Health.

\section{Author details}

'Preveza, Greece. '2Laboratory of Immunoregulation, National Institute of Allergy and Infectious Diseases, National Institutes of Health, 10 Center Drive, Bethesda, MD 20892, USA.

Published: 31 August 2012

\section{References}

1. Li FY, Chaigne-Delalande B, Kanellopoulou C, Davis JC, Matthews HF, Douek DC, Cohen Jl, Uzel G, Su HC, Lenardo MJ: Second messenger role for Mg2+ revealed by human T-cell immunodeficiency. Nature 2011, 475:471-476.

2. Freier S, Kerem E, Dranitzki Z, Schlesinger M, Rabinowitz R, Brautbar C, Ashkirat M, Naparstek Y: Hereditary CD4+ T lymphocytopenia. Arch Dis Child 1998, 78:371-372.

3. Isgro A, Sirianni MC, Gramiccioni C, Mezzaroma I, Fantauzzi A, Aiuti F: Idiopathic CD4+ lymphocytopenia may be due to decreased bone marrow clonogenic capability. Int Arch Allergy Immuno/ 2005, 136:379-384.

4. Hubert P, Bergeron F, Ferreira V, Seligmann M, Oksenhendler E, Debre P, Autran B: Defective p56Lck activity in T cells from an adult patient with idiopathic CD4+ lymphocytopenia. Int Immunol 2000, 12:449-457.
5. Fruhwirth M, Clodi K, Heitger A, Neu N: Lymphocyte diversity in a 9-year-old boy with idiopathic CD4+ T cell lymphocytopenia. Int Arch Allergy Immunol 2001, 125:80-85.

6. Laurence J, Mitra D, Steiner M, Lynch DH, Siegal FP, Staiano-Coico L: Apoptotic depletion of CD4+ T cells in idiopathic CD4+ T lymphocytopenia. J Clin Invest 1996, 97:672-680.

7. Roger PM, Bernard-Pomier G, Counillon E, Breittmayer JP, Bernard A, Dellamonica P: Overexpression of Fas/CD95 and Fas-induced apoptosis in a patient with idiopathic CD4+ T lymphocytopenia. Clin Infect Dis 1999, 28:1012-1016.

8. Zonios DI, Falloon J, Bennett JE, Shaw PA, Chaitt D, Baseler MW, Adelsberger JW, Metcalf JA, Polis MA, Kovacs SJ, Kovacs JA, Davey RT, Lane HC, Masur H,Sereti I: Idiopathic CD4+ lymphocytopenia: natural history and prognostic factors. Blood 2008, 112:287-294.

9. Lee PI, Ciccone EJ, Read SW, Asher A, Pitts R, Douek DC, Brenchley JM, Sereti I: Evidence for translocation of microbial products in patients with idiopathic CD4 lymphocytopenia. J Infect Dis 2009, 199:1664-1670.

10. Malaspina A, Moir S, Chaitt DG, Rehm CA, Kottilil S, Falloon J, Fauci AS: Idiopathic CD4+ T lymphocytopenia is associated with increases in immature/transitional B cells and serum levels of IL-7. Blood 2007, 109:2086-2088.

11. Fry TJ, Connick E, Falloon J, Lederman MM, Liewehr DJ, Spritzler J, Steinberg SM, Wood LV, Yarchoan R, Zuckerman J, Landay A, Mackall CL: A potential role for interleukin-7 in T-cell homeostasis. Blood 2001, 97:2983-2990.

12. Hodge JN, Srinivasula S, Hu Z, Read SW, Porter BO, Kim I, Mican JM, Paik C, Degrange P, Di Mascio M, Sereti I: Decreases in IL-7 levels during antiretroviral treatment of HIV infection suggest a primary mechanism of receptor-mediated clearance. Blood 2011, 118:3244-3253.

13. Puronen CE, Thompson WL, Imamichi H, Beq S, Hodge JN, Rehm C, Parker R, DerSimonian R, Brenchley JM, Sereti I: Decreased interleukin 7 responsiveness of T lymphocytes in patients with idiopathic CD4 lymphopenia. J Infect Dis 2012, 205:1382-1390.

14. Salit RB, Hankey KG, Yi R, Rapoport AP, Mann DL: Detection of CD4(+) T-cell antibodies in a patient with idiopathic CD4 T lymphocytopenia and cryptococcal meningitis. Br J Haemato/ 2007, 139:133-137.

15. Signorini S, Pirovano S, Fiorentini S, Stellini R, Bianchi V, Albertini A, Imberti L: Restriction of T-cell receptor repertoires in idiopathic CD4+ lymphocytopenia. Br J Haematol 2000, 110:434-437

16. Netea MG, Brouwer AE, Hoogendoorn EH, Van der Meer JW, Koolen M, Verweij PE, Kullberg BJ: Two patients with cryptococcal meningitis and idiopathic CD4 lymphopenia: defective cytokine production and reversal by recombinant interferon- gamma therapy. Clin Infect Dis 2004, 39:e83-87.

17. Hernandez PA, Gorlin RJ, Lukens JN, Taniuchi S, Bohinjec J, Francois F, Klotman ME, Diaz GA: Mutations in the chemokine receptor gene CXCR4 are associated with WHIM syndrome, a combined immunodeficiency disease Nat Genet 2003, 34:70-74.

18. Scott-Algara D, Balabanian K, Chakrabarti LA, Mouthon L, Dromer F, Didier C, Arenzana-Seisdedos F, Lortholary O: Idiopathic CD4+ T-cell lymphocytopenia is associated with impaired membrane expression of the chemokine receptor CXCR4. Blood 2009, 115:3708-3717.

19. Kuijpers TW, ljspeert $H$, van Leeuwen EM, Jansen $M H$, Hazenberg MD, Weijer KC, van Lier RA, van der Burg M: Idiopathic CD4+ T lymphopenia without autoimmunity or granulomatous disease in the slipstream of RAG mutations. Blood 2011, 117:5892-5896.

20. Gorska MM, Alam R: A mutation in the human Uncoordinated 119 gene impairs TCR signaling and is associated with CD4 lymphopenia. Blood 2012, 119:1399-1406.

21. Nielsen-Saines K: Idiopathic CD4+ lymphocytopenia. In UpToDate. Waltham, MA: UpToDate Inc:; 2011.

22. Walker UA, Warnatz K: Idiopathic CD4 lymphocytopenia. Curr Opin Rheumatol 2006, 18:389-395.

23. Zonios DI, Falloon J, Huang CY, Chaitt D, Bennett JE: Cryptococcosis and idiopathic CD4 lymphocytopenia. Medicine (Baltimore) 2007, 86:78-92.

24. Bofill M, Janossy G, Lee CA, MacDonald-Burns D, Phillips AN, Sabin C, Timms A, Johnson MA, Kernoff PB: Laboratory control values for CD4 and CD8 T lymphocytes. Implications for HIV-1 diagnosis. Clin Exp Immunol 1992, 88:243-252.

25. Busch MP, Valinsky JE, Paglieroni T, Prince HE, Crutcher GJ, Gjerset GF, Operskalski EA, Charlebois E, Bianco C, Holland PV, Petersen LR, Hollingsworth CG, Mosley JW: Screening of blood donors for idiopathic CD4+ Tlymphocytopenia. Transfusion 1994, 34:192-197. 
26. Rueger MA, Miletic H, Dorries K, Wyen C, Eggers C, Deckert M, Faetkenheuer $\mathrm{G}$, Jacobs AH: Long-term remission in progressive multifocal leukoencephalopathy caused by idiopathic CD4+T lymphocytopenia: a case report. Clin Infect Dis 2006, 42:e53-56.

27. Turett GS, Telzak EE: Normalization of CD4+ T-lymphocyte depletion in patients without HIV infection treated for tuberculosis. Chest 1994, 105:1335-1337.

28. Kirtava Z, Blomberg J, Bredberg A, Henriksson G, Jacobsson L, Manthorpe R: CD4+ T-lymphocytopenia without HIV infection: increased prevalence among patients with primary Sjogren's syndrome. Clin Exp Rheumatol 1995, 13:609-616.

29. Khoruts A, Fraser JM: A causal link between lymphopenia and autoimmunity. Immunol Lett 2005, 98:23-31.

30. Krupica T Jr., Fry TJ, Mackall CL: Autoimmunity during lymphopenia: a twohit model. Clin Immunol 2006, 120:121-128.

31. Mandl T, Bredberg A, Jacobsson LT, Manthorpe R, Henriksson G: CD4+ T-lymphocytopenia--a frequent finding in anti-SSA antibody seropositive patients with primary Sjogren's syndrome. J Rheumatol 2004, 31:726-728.

32. Ferraccioli GF, Tonutti E, Casatta L, Pegoraro I, De Vita S, Sala P, Ravaioli T, Bartoli E: CD4 cytopenia and occasional expansion of CD4+CD8+lymphocytes in Sjogren's syndrome. Clin Exp Rheumatol 1996, 14:125-130.

33. Theander E, Henriksson G, Ljungberg O, Mandl T, Manthorpe R, Jacobsson LT: Lymphoma and other malignancies in primary Sjogren's syndrome: a cohort study on cancer incidence and lymphoma predictors. Ann Rheum Dis 2006, 65:796-803.

34. Sinicco A, Maiello A, Raiteri R, Sciandra M, Dassio G, Zamprogna C, Mecozzi B: Pneumocystis carinii in a patient with pulmonary sarcoidosis and idiopathic CD4+ T lymphocytopenia. Thorax 1996, 51:446-447: discussion 448-449.

35. Sweiss NJ, Salloum R, Gandhi S, Alegre ML, Sawaged R, Badaracco M, Pursell K, Pitrak D, Baughman RP, Moller DR, Garcia JG, Niewold TB: Significant CD4, CD8, and CD19 lymphopenia in peripheral blood of sarcoidosis patients correlates with severe disease manifestations. PLoS One 2010, 5:e9088.

36. Crouser ED, Lozanski G, Fox CC, Hauswirth DW, Raveendran R, Julian MW: The CD4+ lymphopenic sarcoidosis phenotype is highly responsive to antitumor necrosis factor-\{alpha\} therapy. Chest 2010, 137:1432-1435.

37. Cunningham-Rundles C, Murray HW, Smith JP: Treatment of idiopathic CD4 T lymphocytopenia with IL-2. Clin Exp Immunol 1999, 116:322-325

38. Warnatz K, Draeger R, Schlesier M, Peter HH: Successful IL-2 therapy for relapsing herpes zoster infection in a patient with idiopathic CD4+ T lymphocytopenia. Immunobiology 2000, 202:204-211.

39. Wilhelm M, Weissinger F, Kunzmann V, Muller JG, Fahey JL: Idiopathic CD4+ T cell lymphocytopenia evolving to monoclonal immunoglobulins and progressive renal damage responsive to IL-2 therapy. Clin Immuno/ 2001, 99:298-304

40. Yilmaz-Demirdag Y, Wilson B, Lowery-Nordberg M, Bocchini JA Jr., Bahna SL: Interleukin-2 treatment for persistent cryptococcal meningitis in a child with idiopathic CD4(+) T lymphocytopenia. Allergy Asthma Proc 2008, 29:421-424.
41. Trojan T, Collins R, Khan DA: Safety and efficacy of treatment using interleukin-2 in a patient with idiopathic CD4(+) lymphopenia and Mycobacterium avium-intracellulare. Clin Exp Immunol 2009, 156:440-445.

42. Sternfeld T, Nigg A, Belohradsky BH, Bogner JR: Treatment of relapsing Mycobacterium avium infection with interferon-gamma and interleukin-2 in an HIV-negative patient with low CD4 syndrome. Int J Infect Dis 2009, 14 Suppl 3:e198-201.

43. Holland SM, Eisenstein EM, Kuhns DB, Turner ML, Fleisher TA, Strober W, Gallin $\mathrm{Jl}$ : Treatment of refractory disseminated nontuberculous mycobacterial infection with interferon gamma. A preliminary report. NEng/ J Med 1994, 330:1348-1355.

44. Rosenberg SA, Sportès C, Ahmadzadeh M, Fry TJ, Ngo LT, Schwarz SL, StetlerStevenson M, Morton KE, Mavroukakis SA, Morre M, Buffet R, Mackall CL, Gress RE: IL-7 administration to humans leads to expansion of CD8+ and CD4+ cells but a relative decrease of CD4+ T-regulatory cells. J Immunother (1997) 2006, 29:313-319.

45. Sereti I, Dunham RM, Spritzler J, Aga E, Proschan MA, Medvik K, Battaglia CA, Landay AL, Pahwa S, Fischl MA, Asmuth DM, Tenorio AR, Altman JD, Fox L, Moir S, Malaspina A, Morre M, Buffet R, Silvestri G, Lederman MM; ACTG 5214 Study Team: IL-7 administration drives T cell-cycle entry and expansion in HIV-1 infection. Blood 2009, 113:6304-6314.

46. Levy Y, Lacabaratz C, Weiss L, Viard JP, Goujard C, Lelièvre JD, Boué F, Molina JM, Rouzioux C, Avettand-Fénoêl V, Croughs T, Beq S, Thiébaut R, Chêne G, Morre M, Delfraissy JF: Enhanced T cell recovery in HIV-1-infected adults through IL-7 treatment. J Clin Invest 2009, 119:997-1007.

47. Interleukin-7 (CYT107) Treatment of Idiopathic CD4 Lymphocytopenia: Expansion of CD4 T cells (ICICLE) [http://clinicaltrials.gov/ct2/show/NCT008 39436?term=cyt107+trial\&rank=9]

48. Patel A, Patel J, Ikwuagwu J: A case of progressive multifocal leukoencephalopathy and idiopathic CD4+ lymphocytopenia. J Antimicrob Chemother 2010, 65:2697-2698.

49. Petersen EJ, Rozenberg-Arska M, Dekker AW, Clevers HC, Verdonck LF: Allogeneic bone marrow transplantation can restore $C D 4+$ T-lymphocyte count and immune function in idiopathic CD4+ T-lymphocytopenia. Bone Marrow Transplant 1996, 18:813-815.

50. Cervera C, Fernández-Avilés F, de la Calle-Martin O, Bosch X, Rovira M, Plana M, Moreno A, García F, Miró JM, Martínez A, Gallart T, Carreras E, Blade J, Gatell $J$ M: Non-myeloablative hematopoietic stem cell transplantation in the treatment of severe idiopathic CD4+ lymphocytopenia. Eur J Haematol 2011, 87:87-91.

doi:10.1186/ar4027

Cite this article as: Zonios D, et al.: Idiopathic CD4 lymphocytopenia: a case of missing, wandering or ineffective T cells. Arthritis Research \& Therapy 2012, $14: 222$ 